\title{
Molecular profiling is not the future: it is now!
}

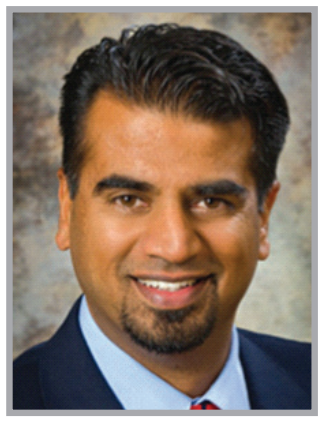

Bobby Reddy* speaks to Gemma Westcott, Commissioning Editor: Dr Reddy graduated from the UCLA School of Medicine in 1996. Shortly after, he obtained an internship and did his residency in Internal Medicine at Harbor UCLA Medical Center. He then went on to do his fellowship in Hematology and Oncology at City of Hope. Since then, he has been working in private practice (full and part time) for the past 11 years and has had an academic appointment as teaching faculty at Harbor UCLA. Prior to his current role, Dr Reddy worked as a senior medical director as Caris Life Sciences.

\section{Q What was it that sparked your interest in cancer research?}

Too many patients succumb to cancer despite intensive research efforts. I think there has to be a better way forward, and we are at a unique time when new technologies have put the answers within our grasp. Who would not want to be a part of that? In addition, my father was a research scientist, a PhD in biochemistry, and so I was indoctrinated at an early age, spending many summers in his laboratory.

Q Can you tell our readers how you came to be in your current role?

I had worked as a senior medical director at Caris for 2 years prior to my current role. The rapid pace of development in the cancer space beyond genomics, now to 'panomics,' caused me to consider pulling back further from clinical time to pursue this opportunity. I still see patients one clinic day per week, which was the compromise that allowed me to translate the bench science into clinically actionable results for patients.

\section{Q What research does Caris Life Sciences ${ }^{\circledR}$ focus on \& what are Caris's biggest achievements?}

The idea that personalized medicine and precise information can improve healthcare for everyone is what drives our work at Caris Life Sciences. Our research focuses on providing evidence to physicians that Caris Molecular Intelligence (CMI) will benefit their patients. We do that by demonstrating that clinically actionable molecular targets can be identified in the majority of patients and that treating patients based on the CMI profile can result in improved outcomes.

We published several manuscripts and abstracts in 2014 illustrating the frequency of actionable targets in various cancer types including glioblastoma, ovarian, bladder and triple-negative breast cancer.

*Caris Life Sciences, 6655 N MacArthur Blvd, Irving, TX 75039, USA

\section{KEYWORDS}

- Caris Life Sciences • cancer biomarkers $\bullet$ cancer research - molecular profiling • luminal breast cancer • male breast cancer $\bullet$ PD-1

- personalized medicine $\bullet$ PD-L1

- precision medicine $\bullet$ SABCS • San Antonio Breast Cancer Symposium - triple-negative breast cancer • tumor profiling

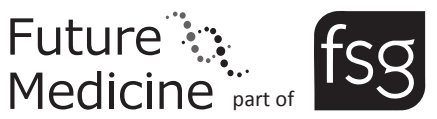


By correlating the tumor's unique biomarker information with biomarker-drug associations supported by evidence in clinical studies, we are able to provide guidance to treating oncologists about which treatments are most likely to be effective and which treatments are most likely to be ineffective for each patient. Our research primarily focuses on rare and aggressive cancers and refractory disease, where physicians have little to no guidance once conventional treatment option(s) have been exhausted.

Our biggest achievement, from a research perspective, was the publication this year of a study in metastatic breast cancer showing that CMI resulted in improvement in progressionfree survival in $44 \%$ of tested patients, relative to the progression-free survival on their previous therapy.

Q The company presented two studies at the 2014 San Antonio Breast Cancer Symposium (9-13 December 2014), both of which utilized comprehensive molecular profiling technologies. Why is tumor profiling important?

Comprehensive tumor profiling is critically important as it helps guide the oncologist to drugs that may be effective or ineffective for a specific patient. There are some studies that estimate that after initial treatment fails, as many as $95 \%$ of patients with cancer will not respond to the next treatment suggested by conventional methods. By understanding the unique genetic and molecular characteristics of a patient's disease through comprehensive tumor profiling, physicians can develop regimens designed for maximum effectiveness, which helps the patient avoid the high cost and toxicity of treatments that are less likely to work. Using these innovative tools is not only good clinical practice, often leading to better patient outcomes, but also more cost-efficient, as the expense of the screening or testing is generally much less expensive than the cost of ineffective treatment.

Q The first study featured the findings of Caris's HER2-positive luminal breast cancer research. Can you tell us about that?

Luminal breast cancers are hormone-receptorpositive tumors and have a better prognosis than nonhormonal tumors. However, if a luminal breast cancer patient does have a recurrence, treatment options need to be identified. New drugs targeting the immune checkpoint pathway are having success in clinical trials and we wanted to explore the presence of possible predictive markers for these drugs in this subtype of breast cancer. We found that significant numbers of luminal breast cancers did express PD-1 and PD-L1, two targets of certain immune checkpoint inhibitors, however, when you look at the HER2-positive subgroup of luminal cancers, only one patient expressed these markers.

\section{Q What will the results mean for luminal breast cancer patients?}

As immune checkpoint therapy continues to gain momentum in other areas of oncology, this study shows that the relative lack of PD-L1 and PD-1 expression in the HER2-positive cohort indicates a lesser likelihood of benefit from immune checkpoint therapy in this subpopulation compared with patients with triple-negative breast cancer. The expression of immune regulatory targets in this cohort suggests that targeted therapies that inhibit immune checkpoints such as PD-1, PD- L1, CTLA4, B7-H3 and IDO1 may be especially effective in patients with luminal breast cancers with ER-positive and HER2-negative disease.

Q The second presentation featured research on male breast cancer patients. Could you also tell us about that?

We evaluated 60 male and 5000 female breast cancer samples to identify differences and commonalities between the two diseases. Specifically, we looked for patterns within the male breast cancer (MBC) cohort that might show relationships with known subtypes such as TNBC, HER2-positive and ER-positive. We were also looking for novel treatment options and were able to identify possible therapies in $98 \%$ of MBC cases based on protein expression and gene copy number. Very few clinically actionable mutations were found. Other findings included:

Overexpression of androgen receptor (70\%) in MBC patients, warranting continued investigation of antiandrogen therapies in this population, especially in combination with other hormone replacement therapies, due to the coincidence of ER, PR and androgen receptor in $50 \%$ of cases;

HER 2 overexpression and amplification were lower in MBC samples than in female breast cancer (FBC); however, the investigators noted that when HER2 aberrations are identified in 
MBC patients, use of HER2-targeted therapies may be efficacious;

The PI3K pathway (PIK3CA mutation or loss of PTEN) was aberrated in $50 \%$ of MBC samples, suggesting that antihormonal therapies may be warranted, especially in combination with PI3K pathway inhibitors;

In total, $80 \%$ of $\mathrm{MBC}$ cases tested for $\mathrm{Ki}-67$, a protein associated with aggressive disease, were high. Similar to FBC, $83 \%$ of MBC cases that were high for $\mathrm{Ki} 67$ also overexpressed androgen receptor.

Q What will these significant differences in tumor profiles mean for MBC patients?

The study in $\mathrm{MBC}$ reflects an expanding range of potential treatment options for this patient population. The data from this rare cancer population underscore the importance of examining the individual molecular profile of a patient's cancerous tissue to more clearly delineate the most appropriate therapy for that patient, which may incorporate standard or investigational treatment options. The differences we observed in gene mutation, amplification and protein expression profiles suggest that the standard of care in female breast cancer patients may not necessarily be the best treatment option for male breast cancer patients.

Q Finally, what lies on the horizon for breast cancer research at Caris Life Sciences?

We are going to continue to be active in breast cancer research in 2015. We will soon be publishing a manuscript characterizing actionable biomarkers in triple-negative breast cancer. We also expect to have some retrospective outcomes data this year demonstrating how patients responded to various CMI-directed therapies.

\section{Disclaimer}

The opinions expressed in this interview are those of the interviewee and do not necessarily reflect the views of Future Medicine Ltd.

\section{Financial \& competing interests disclosure}

$B$ Reddy discloses stock ownership and employment by Caris Life Sciences. B Reddy has no other relevant affiliations or financial involvement with any organization or entity with a financial interest in or financial conflict with the subject matter or materials discussed in the manuscript apart from those disclosed.

No writing assistance was utilized in the production of this manuscript. 